\title{
Open surgical revascularization of chronic total occlusion of the infrarenal aorta
}

\author{
Dragan Piljic ${ }^{*}$, Mate Petricevic ${ }^{2}$, Dilista Piljic ${ }^{1}$, Gordan Galic ${ }^{3}$, Mustafa Tabakovic ${ }^{1}$, Alen Hajdarevic ${ }^{1}$, Nail Sehic ${ }^{1}$, Tarik Bakalovic ${ }^{1}$, \\ AmarSkakic $^{1}$, Mirza Tokic ${ }^{1}$, Fahrudin Sabanovic ${ }^{4}$ and Almir Kusturica ${ }^{1}$ \\ ${ }^{1}$ University Clinical Center Tuzla, Cardiovascular Surgery Clinic, Tuzla, Bosnia and Herzegovina \\ ${ }^{2}$ University Hospital Center Zagreb, Zagreb, Croatia \\ ${ }^{3}$ University Hospital Center Mostar, Bosnia and Herzegovina \\ ${ }^{4}$ Health Care Center Zenica, Bosnia and Herzegovina
}

\begin{abstract}
We report a case of chronic total occlusion of the aorta with critical limb ischemia (CLI) of the lower limbs due to chronic total occlusion (CTO) of infrarenal aorta and extensive bilateral iliac disease. This case was treated by open surgical revascularization, after unsuccessful attempt of percutaneous endovascular stenting treatment.
\end{abstract}

\section{Case presentation}

A 46-year-old male, with a history of hypertension and peripheral vascular disease (including previous unsuccessful attempt endovascular stenting) was referred to our hospital for conventional open surgical treatment. The patient underwent a computed tomographic angiography, which showed complete occlusion of the infrarenal abdominal aorta, as well as both common and external iliac artery (Figure 1). He was a heavy smoker (60 cigarettes/day) and had an untreated hyperlipidemia.

The operation was performed using the transperitoneal approach with limited thrombectomy through infrarenal aortotomy without transecting the aorta.

The patient underwent aortic bifemoral revascularisation, with placement of a Dacron bifurcation graft of $12 / 6 \mathrm{~mm}$. The Piljic - method was used (restricted intraoperative and postoperative fluid regime and mini-laparotomy, including surgical approach trough 8 to $10 \mathrm{~cm}$ paraumbilical incision. The small and large bowels were retracted to the side without being elevated out of the abdominal cavity) [1]. The patient was transfered to the intensive care unit following successful surgical repair. The patient was transfered to the department of cardiovascular surgery on the postoperative day one.

Uneventful postoperative recovery resulted in hospital discharge on postoperative day four. Postoperative CTA with contrast showed a neat flow (Figure 2).

Patient postoperative period has been followed up for 12 months, which ended with satisfactory general clinical and local state of both legs.

\section{Discussion}

In patients presenting with aortoiliac occlusive disease (AIOD), the total occlusion of the infrarenal aorta has been seen in 3 to $8.5 \%$ of cases [2].

Common causes of chronic infrarenal aortic occlusion (CIAO) include: a) atherosclerotic occlusive disease; b) middle aortic syndrome; c) Takayasu arteritis; d) fibromuscular dysplasia; e) neurofibromatosis; and f) coral reef aorta [2]. Although standardized infrarenal aortobifemoral bypass (AoBFB) remains the surgical procedure of choice for CIAO, operative decisions may proceed beyond AoBFB in complicated cases. Different therapeutic strategies include axillo-(bi) femoral bypass (AxBFB), aortoiliac endarterectomy (AIE), or hybrid procedures. AxBFB grafting usually refers to patients of high risk for aortic clamping, or patients with many comorbidities that prohibit an extensive transperitoneal procedure [3]. Surgical management
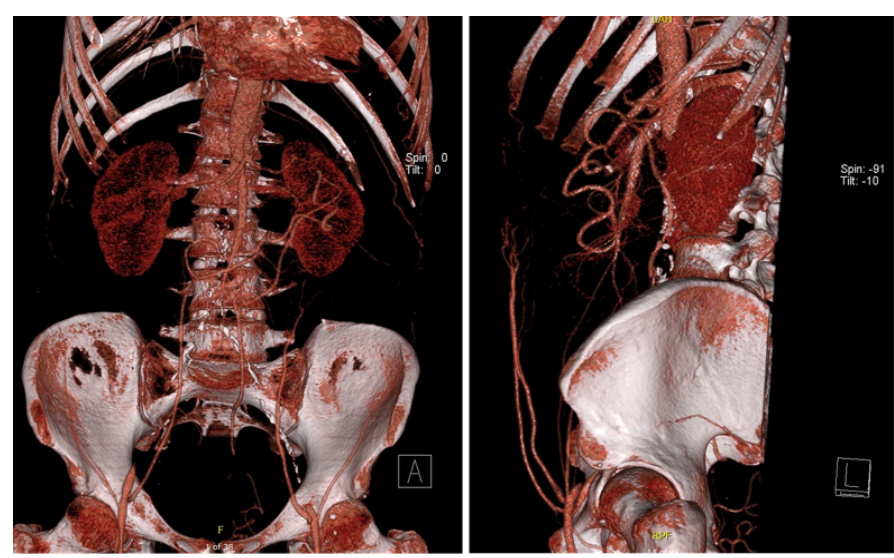

Figure 1. Computed tomographic angiography showed complete occlusion of the infrarenal abdominal aorta and both common and external iliac arteries. A lateral view showed collateral blood flow from internal thoracic arteries through subcutaneous epigastric abdominal vessels to the common femoral arteries

${ }^{\star}$ Correspondence to: Dragan Piljic, M.D., Ph.D, Department of Cardiovascular Surgery, University Clinical Center Tuzla, Bosnia and Herzegovina, Tel: +38735303202; E-mail: dragan.piljic@dr.com

Received: November 11, 2019; Accepted: November 25, 2019; Published: November 28, 2019 

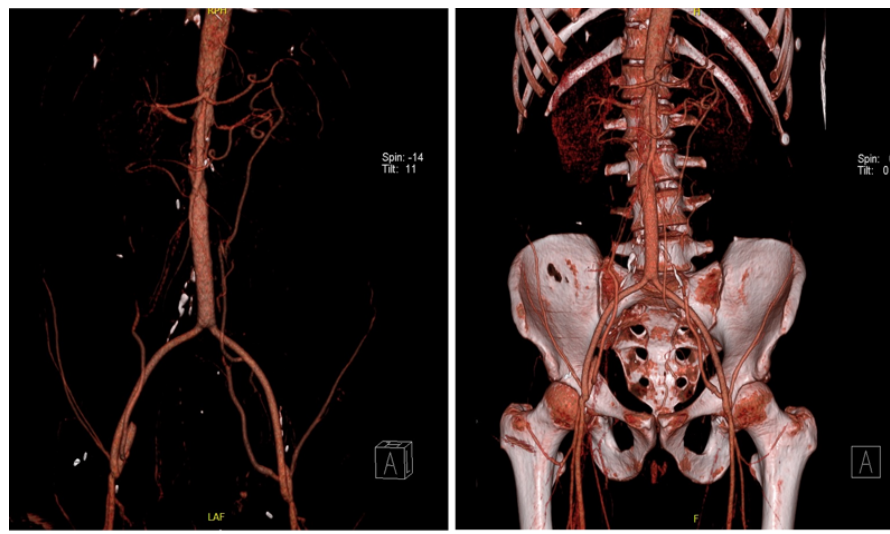

Figure 2. Postoperative CTA with contrast showed a neat flow

of the totally occluded abdominal aorta is highly complex. Surgical intervention is beneficial for patients with totally occluded aorta, even if ischemic complaints are relatively mild and stable [4].

We report a case of chronic total occlusion of the aorta with critical limb ischemia (CLI) of the lower limbs due to chronic total occlusion (CTO) of infrarenal aorta and extensive bilateral iliac disease. This case was treated by open surgical revascularization, after unsuccessful attempt of percutaneous endovascular stenting treatment.

\section{Conclusion}

Open surgical recanalization of aortic occlusion in a patient with previously unsuccessful attempt endovascular stenting is feasible and can be life-saving. Use of open surgical revascularisation can result in rapid clinical recovery and lower mortality and morbidity.

\section{Informed consent}

The patient provided written informed consent for publication of the figures.

\section{Declaration of conflict of interest}

The authors declare that there is no conflict of interest.

\section{Funding}

This work received no specific grant from any funding agency within public, commercial, or not-fot-profit sectors.

\section{References}

1. Piljic D, Petricevic M, Piljic D, Ksela J, Robic B, et al. (2015) Restrictive versus Standard Fluid Regimen in Elective Minilaparotomy Abdominal Aortic RepairProspective Randomized Controlled Trial. Thorac Cardiovasc Surg 64: 296-303.

2. Shah M, Patnaik S, Sinha R, Opoku-Asare I, Chaudhry K, et al. (2017) Revascularization of Chronic Total Occlusion of the Infrarenal Aorta in a Patient with Triple Vessel Disease: Report of a Case Treated by Endovascular Approach. Case Rep Cardiol 2017: 7983748.

3. Indes JE, Pfaff MJ, Farrokhyar F, Brown H, Hashim P, et al. (2013) Clinical outcomes of 5358 patients undergoing direct open bypass or endovascular treatment for aortoiliac occlusive disease: a systematic review and meta-analysis. J Endovasc Ther 20: $443-$ 455. [Crossref]

4. Illuminati G, Calio FG, Mangialardi N, Bertagni A, Vietri F, et al. (1996) Results of axillofemoral by-passes for aorto-iliac occlusive disease. Langenbecks Arch Chir 381: 212-217.

5. Mavioglu I, Veli Dogan O, Ozeren M, Dolgun A, Yucel E (2003) Surgical management of chronic total occlusion of abdominal aorta. J Cardiovasc Surg 44: 87-93.

Copyright: (2019 Piljic D. This is an open-access article distributed under the terms of the Creative Commons Attribution License, which permits unrestricted use, distribution, and reproduction in any medium, provided the original author and source are credited. 